\title{
Blockchain at the Service of Ocean Challenges: Discard Management and Bycatch Control
}

\author{
Hossein Noroozi ${ }^{1}$, Afshin Abolhasani ${ }^{2}$ \\ (Received: 02 January 2021 / Revised: 09 January 2021 / Accepted: 14 January 2021)
}

\begin{abstract}
-the purpose of this paper is to introduce a new alternative method of seafood management by using blockchain technology with a particular emphasis on bycatch and overfishing. The paper has been investigated through an innovative connection between the various consumers in the world, what we call bycatch can be considered as food for humans, animals, or plants. In addition, numerous parties that are directly or indirectly connected to the seafood industry can utilize the blockchain platform in order to facilitate seafood trading, especially what is called bycatch. The data analyzing in this paper has been clarified using a decentralized management system, seafood resources, and human consumption can stay in a semi-equilibrium. Furthermore, the paper can take the first steps to reduce environmental catastrophes caused by humans on the ocean.
\end{abstract}

Keywords-blockchain, bycatch, decentralized management, oceans, seafood.

\section{INTRODUCTION}

A life depends on a healthy world, but human activities endanger the natural environment's interwoven structures of atmosphere, seas, watercourses, soil, ice cover, and biosphere [1]. Oceans provide essential natural resources such as food, medicine, biofuels, and other products. The livelihoods of over three billion people are dependent on marine and coastal biodiversity. The sea provides and regulates our rainwater, drinking water, weather, environment, coastlines, most of our food, and even the oxygen in the air we breathe [2]. Marine Protected Areas also help to alleviate poverty by increasing fish catches and jobs, as well as improving health [2-4].

As far as we know, there are numerous disasters in the oceans such as overexploitation, marine litter, marine pollution, and some more related to seafood. One of the most important issues is bycatch and discards and lack of efficient ocean management. Non-target fish and ocean animals are captured as bycatch, including what is carried to port and what is dumped at sea, dead or dying [4-7]. Bycatch is one of the most serious threats to the fisheries, contributing to overfishing and a sharp reduction in fish stocks. Since bycatch is largely unknown, it has been a chronic problem for decades.

Hossein Noroozi is with Department of Sociology, University of Milano-Bicocca, Milan, 20126, Italy. E-mail: h.noroozi@campus.unimib.it

Afshin Abolhasani is with Department of Environmental and Earth Science, University of Milano- Bicocca, Milano, 20126, Italy. E-mail: a.abolhasani@campus.unimib.it
During the 1990s, bycatch claimed the lives of 650,000 whales, dolphins, and seals all over the world. Between 17 and 22 percent of fish captured in the US, fisheries are discarded before reaching shore, amounting to roughly 2 billion pounds per year [7-8].

In recent years, commercial fisheries have brought in about 160 billion pounds of marine catch per year around the world, equating to nearly 400 million pounds a day. According to recent figures, up to $40 \%$ of the global catch is discarded overboard. Everything can be caught as bycatch, including dolphins encircled to bring you canned tuna, sea turtles caught to bring you shrimp, flounder are thrown overboard to put seared scallops on the menu, endangered whales migrating thousands of miles just to become entangled for the sake of lobster bisque, and millions of pounds of halibut or cod that are wasted after fishermen have already reached the limit. Most of the captured wildlife is thrown overboard, dead or dying, as waste. However, we know the types of problems and we can predict the extent of contamination, we still don't have any system which can give us the updated information based on real data which we can trust, and this is what are focusing [9-12].

Equilibrium between three principal aspects of the seafood industry including human consumption, oceans, and economic growth is crucially important for humans. Oceans, waters, and aquatic resources have a two-way relationship with economic development. While an increase in human well-being often comes at the expense of ecological integrity, new alternative development methods to mitigate negative anthropogenic impacts on the marine environment could emerge [14-16]. For example, we can change production and consumption 
habits, increase global knowledge, and monitor human

sustainable resource use. activities through decentralized management and

\section{Seafood}

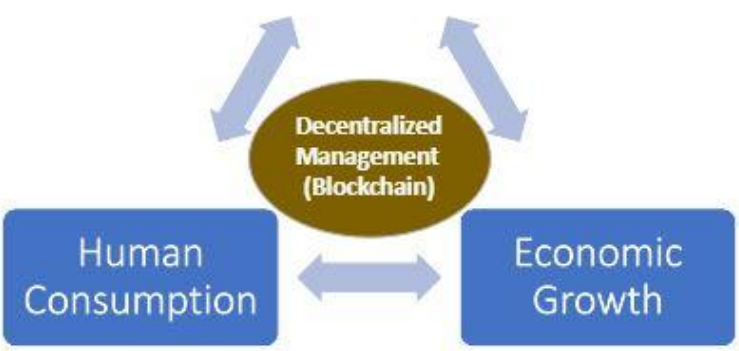

Figure. 1. Decentralized blockchain management.

Without concerted efforts, marine ecosystem disasters are expected to increase by 2050 . To prevent overfishing, marine protected areas must be wellmanaged and resourced, and new legislation must be implemented. Decentralized management and continuous sustainability in the open ocean and deep-sea areas can only be accomplished by the expanded international collaboration to protect fragile ecosystems as shown in Figure 1.

\section{METHOD}

\section{A. Data, the basis of the resource management}

Human activities are rapidly endangering, degrading, or destroying oceans, seas, and marine resources, reducing their capacity to provide essential ecological services and posing new challenges. At this is stage some key questions arise:

- How can we make a balance between oceans resources, economic growth, and human consumption?

- How can we manage and protect the marine environment?

- Can New technologies help policy-makers in environmental management?

We investigate the decentralized management which Blockchain is based on, is bidding to keep the seafood industry sustainable through an incentivized and transparent global database that could improve resource management. Therefore, from a data analytics viewpoint, lack of information and unclear information are two main challenges against control and managing environmental disasters in the oceans.

1) Lack of information: With an in-depth look at the history of ocean disasters, we can understand so many disasters that happened because of unclear information or lack of knowledge. The biggest ship (The Titanic) in the world in 1912 sank in the ocean because of insufficient knowledge. Recently, the same history on a larger scale is happening in the ocean environment. There is a correlation between the amount of knowledge, the size of the task, and the greatness of the result. , less information and knowledge for performing big tasks results in a big disaster. Therefore, with minimum knowledge about the oceans, we are currently taking big actions which means that we have to expect big disasters. There are just a few countries that are studying life below water and they are usually able to focus on studying the marine environment nearby. Without global awareness and sufficient management, the information that is gathered by scientists and just usually archived by journals, cannot impact the current phenomenon. Thus, the problem remains and probably gets worse.

2) Unclear information: After the industrial revolution and inventing new technologies, many companies have been established and by technological and economic power invented bigger, faster, and powerful tools to extract more resources from the environment. Recent scientific researches show we are going to withdraw more than the environment capacity. We argue, two main groups are not interested in clear information. First, the people who are gaining profits by over-extracting from the environment. The second group is those who don't want to decrease their facilities in their life. When we talk about unclear information, it means that we don't know exactly how much of the resources have been extracted? How many resources exist? What is the process of producing the final product? And what is the fate of that product? How much pollution is 
produced from the starting point until being consumed by people?.

How can we think about protecting the environment if we don't have clear information about the mentioned questions? Imagine a large urban executive system, decide to reduce the pollution in the city; the first step is to detect the type of pollution and the amount of pollution in order to choose practical solutions and tools to act. On a larger scale, we need to follow the same process. However, we know the usual types of pollution and we can predict the extent of contamination, we still don't have any system which can give us the updated information based on real data.

\section{RESULTS AND DISCUSSION}

\section{A. Decentralized model of bycatch management:}

A recent Globescan survey found that $70 \%$ of consumers want to know more about the sustainability of the seafood they eat such as Figure 2. The paper proposed research project aims to create a global decentralized database for the seafood industry's longterm security and sustainability. The project aims to keep the seafood industry afloat by creating a decentralized global database with incentives to enhance resource management. This concept proposes to manage this by enhancing data collection and sharing through the use of decentralized ledger technology. The paper contend that the majority of ocean industries are using data to improve "efficiency and innovation," and that seafood is lagging behind in this regard. According to the hypothesis, the seafood industry would be incentivized to collect and input data at any point in the supply chain by using this innovative blockchain system; as a result, the entire industry would benefit from a data pool that is conducive to a healthier environment. The paper argue that having access to clear information is the basis of sustainable development. Therefore, if the paper have the information from the starting point (fishery) to the end which means consuming by customers and discarding them as trash can be considered as the first step for emerging different branches of economic activities and protecting the environment then can result in sustainable development. The paper can divide the whole procedure into four division:

1. Fishery

2. Processors

3. Customers

4. Discarding

The process of consuming seafood involving these four divisions. They influence the economy and environment directly. What the paper are suggesting is to create a decentralized network to track the information of the seafood from the starting point until their final destination. Also connecting the four divisions to facilitate the procedure. Separate supply chain players keep information in silos, making it almost impossible to fully or efficiently track a seafood commodity from its source to its final destination. The paper analytics data shows there are several parties involved in the construction of the seafood industry including fishermen, companies, and customers that are connected to this industry all over the world. Additionally, some amount of fishes and other marine animals are discarded at different levels; for example, the discarding of bycatch after fishing, discarding of fishes after processing the seafood and discarding the remaining seafood by customers (figure 2).



Figure. 2. A decentralized network to track the information of the seafood.

B. Distinctive aspects and innovative character of the project:
The seafood that is discarded at different levels is potentially valuable. Minimally they are some organic materials that can be used as nutrients and fertilizers for 
agriculture or they can be used as food for the animals such as animals that are kept in the zoo. The paper claim, through the creation of a decentralized platform we can show the type and amount of waste at different levels. Subsequently, there will be customers who can use the waste as a precursor for their industry. Even in the absence of customers, the paper can collect data for scientists in order to analyze the situation. Therefore, there will be more chances to manage marine biological resources. Some main innovative characters of the proposed project are as follow:

1) Eliminating the brokers: The paper argue that having this network can remove the brokers that can cause decreasing the price. In addition, fewer brokers are equal to consuming less energy. Using less energy is equal to producing less pollution and less negative impact on the environment.

2) Managing the discards: What is considered as discard of seafood can be managed efficiently by using this network. Discards of seafood may happen from fishery to consumption. Therefore, the fisherman can sell the fishes they caught to different processors that can result in fewer discards. Also, it would have good effects on the environment.

3) Efficient marketing and discovering the potentials: This network connects the different divisions and can facilitate trading the seafood and finding suitable destinations. The different divisions can discover new potentials.

4) Scientific data: The traceability of the information of the seafood can help the scientists to analyze the data of the seas and oceans in order to recognize the current condition of the LIFE BELOW WATER that leads to the efficient management of the living organisms.

5) Humanity concerns: People want to know the story behind their seafood. The system can bring customers clear information about seafood that can help them to make sustainable decisions and select superior options.

6) Facilitating the bureaucratic process: The licenses can be issued through the network that can provide clear information regarding the target divisions. It can be useful for the government and the different divisions to spend less time and energy issuing and receiving the licenses. In addition, it can provide the scientists to study the process, the types of the licenses, the numbers of licenses, and related data.
7) Interlacing economy and environment: Currently there is a strict border between economy and environment. If companies want to protect the environment, they would earn less profit. By using the proposed network, the equilibrium between economic growth and environmental challenges in the oceans can be increased.

8) Authenticated data: Although "garbage in, garbage out" is still a problem, it's important to remember that fraud and malfeasance, as well as honest mistakes, are infinitely more observable in a blockchain scenario than in a centralized database scenario. Furthermore, the blockchain's permanence allows for instantaneous auditing of a historical record, which may aid in quickly identifying trends of ongoing fraud, enabling markets to escape risk and regulatory authorities to quickly detect and resolve such instances.

\section{CONCLUSION}

Recently the concepts and issues related to marine management are raised. Therefore, despite difficulties, it is crucial to create a global transparent platform in the line with ocean management for the benefit of policymakers, stakeholders, consumers, and environmental issues. Although, the challenges involved in the decentralized system to ensure effective sustainable development aren't examined.

The proposed model is focusing on discard management and bycatch control through a transparent and tamper-free system like Blockchain. Therefore, what we claim is to connect different parties that are directly or indirectly connected to the seafood industry, in order to facilitate trading seafood, especially what is called bycatch. In addition, an online monitoring system collects real-time data from different parties. Consequently, there would be a chance to manage marine resources more efficiently.

The argue, global access to data can pave the road for companies all over the world to trade their products, especially their seafood waste can add some profits to their business. Moreover, the paper predict that implementing the system regionally can go viral because it can make some profits for different companies in different locations in the world. Finally, the paper believe that data is the basis of sustainable development. without data, researcher can develop but cannot sustain the development.

The proposed model and interventions should preferably be planned and implemented in an integrated, cross-sectoral, and cross-scale manner, in accordance with the ecosystem approach, and with the participation 
of all stakeholders. A shift in how humans perceive, handle and use oceans, seas, and marine resources would be needed to preserve the quality of life that the oceans have given to humanity while also preserving the integrity of their habitats.

\section{REFERENCES}

[1] World Meteorological Organization, 2010; Climate carbon and coral reefs; WMO-No. 1063.

[2] Deloitte Insights 2020; Deloittes 2020 global blockchain survey; from promise to reality.

[3] Oceans environment; available at National Geography: https://www.nationalgeographic.com/environment/oceans/

[4] Implication of blockchain in data science; available at Itproportal: https://www.itproportal.com/features/implications-of-blockchainin-data-science/

[5] Environmental and health impact of shipping seriously underestimated; available at Transport and Environment: https://www.nationalgeographic.com/environment/oceans/

[6] Goal 14: Conserve and sustainably use the oceans, seas and marine environment; available at UN sustainable development goals: https://www.un.org/sustainabledevelopment/oceans/

[7] Environment; available at World Meteorological Organization: https://public.wmo.int/en/our-mandate/focus-areas/environment

[8] IMO and sustainable development goals; available at International Marine

Organization: https://www.imo.org/en/MediaCentre/HotTopics/Pages/Sustainabl eDevelopmentGoals.aspx

[9] What would a clean cruise industry look like? available at Friends of the earth: https://www.imo.org/en/MediaCentre/HotTopics/Pages/Sustainabl eDevelopmentGoals.aspx

[10] Know seafood to launch online consumer seafood market using blockchain; available at Ledger Insights: https://www.ledgerinsights.com/knowseafood-consumer-seafoodmarketplace-blockchain/

[11]Environmental law; available at International Union for Conservation of Nature: https://www.iucn.org/theme/environmental-law

[12]Economics: National Oceans Watch; available at: https://coast.noaa.gov/digitalcoast/data/enow.html

[13] Seafood traceability powered by blockchain; available at Fishcoin: https://fishcoin.co/

[14] Overfishing and pollution have trashed the Mediteranian; available at Oceana Org: https://oceana.org/blog/overfishing-and-pollutionhave-trashed-mediterranean

[15]Can environmentalists use the data to save the environment? available at Smart Data Collective: https://www.smartdatacollective.com/environmentalists-use-bigdata-save-environment/

[16] New blockchain project has potential to revolutionise the seafood industry; available at Panda Org: https://wwf.panda.org/?320232/New-Blockchain-Project-hasPotential-to-Revolutionise-Seafood-Industry 